\title{
The coverage problem for loitering Dubins vehicles
}

\author{
Ketan Savla \\ Francesco Bullo \\ Emilio Frazzoli
}

\begin{abstract}
In this paper we study a facility location problem for groups of Dubins vehicles, i.e., nonholonomic vehicles that are constrained to move along planar paths of bounded curvature, without reversing direction. Given a compact region and a group of Dubins vehicles, the coverage problem is to minimize the worst-case traveling time from any vehicle to any point in the region. Since the vehicles cannot hover, we assume that they fly along static closed curves called loitering curves. The paper presents circular loitering patterns for a Dubins vehicle and for a group of Dubins vehicles that minimize the worst-case traveling time in sufficiently large regions. We do this by establishing an analogy to the disk covering problem.
\end{abstract}

\section{INTRODUCTION}

One of the prototypical missions for Uninhabited Aerial Vehicles, e.g., in environmental monitoring, security, or military setting, is wide-area surveillance. A low-altitude UAV in such a mission must provide coverage of a certain region and investigate events of interest ("targets") as they manifest themselves. In particular, we are interested in cases in which close-range information is required on targets detected by high-altitude aircraft, spacecraft, or ground spotters, and the UAVs must proceed to the location of the detected targets to gather on-site information.

Variations of problems falling in this class have been studied in a number of papers in the recent past, e.g., see [1], [2], [3], [4]. In these papers, the problem is set up in such a way that the location of targets is known a priori and a strategy is computed that attempts to optimize the coverage cost of servicing the known targets. Coordination algorithms for distributed sensing task were proposed and analyzed in [5]. A limitation of the results presented in [5] is the fact that omni-directional or locally controllable vehicles were considered in the problem formulation. Because of this assumption, the results are not applicable to many vehicles of interest, such as aircraft and car-like robots.

In [6] we presented the results of our work for designing closed tours through a set of given points for a nonholonomic vehicle that is constrained to move along planar paths of bounded curvature, without reversing direction. This model is also known as the Dubins vehicle in literature [7]. Path planning for groups of Dubins vehicles has gained considerable interest in recent past [8], [9].

In contrast to simpler vehicles [5] which can wait at a single location while they are idle, Dubins vehicles have

This material is based upon work supported in part by AFOSR MURI Award F49620-02-1-0325.

Ketan Savla and Francesco Bullo are with the Center for Control, Dynamical Systems and Computation, University of California at Santa Barbara, ketansavla@umail.ucsb.edu, bullo@engineering.ucsb.edu

Emilio Frazzoli is with the Aeronautics and Astronautics Department, Massachusetts Institute of Technology, frazzoli@mit.edu to loiter while they are waiting for targets to appear in the region. As a consequence, we need to characterize the configuration of the vehicles at the appearance of new targets in terms of Dubins paths, that we will call loitering patterns.

The main contributions of this paper are as follows. First, we study the reachable set of Dubins vehicle and characterize some of its properties that are particularly useful for the problem at hand. Most importantly, we introduce a certain "covering problem" where a circle or a sector with given parameters is to be contained in the Dubins reachable set of minimal time. Second, we characterize optimal circular loitering for a single Dubins vehicle by exploiting the rotational symmetry of the problem and the simple-connectedness of the Dubins reachable set. Third, we design efficient circular loitering patterns for a single team of multiple Dubins vehicle and provide a bound on the achievable performance for sufficiently large environments. Finally, we consider the case of multiple teams composed of the same number of vehicles. We propose a computational approach to computing loitering patterns based on (1) partitioning the environment into Voronoi partitions generated by virtual centers, (2) moving the virtual centers in such a way as to solve a minimumradius disk-covering problem, and (3) designing efficient loitering patterns for each team in its corresponding Voronoi cell.

The paper is organized as follows. In Section II, we setup the problem and introduce notations that will be used in the rest of the paper. Section III carries a discussion on the reachable set of the Dubins vehicle. The single vehicle and the single team case are considered in Sections IV and V, respectively. In Section VI, we consider the multiple uniform team case. Finally, we conclude with a few remarks about future work in Section VII. Due to lack of space, the proofs are omitted are in this paper; they can found in [10].

\section{Problem Setup and notations}

In this section we setup the main problem of the paper and review some basic required notation.

\section{A. Dubins vehicle and feasible path}

A Dubins vehicle is a planar vehicle that is constrained to move along paths of bounded curvature, without reversing direction and maintaining a constant speed. We will design loitering patterns for $n$ Dubins vehicle in a compact region $\mathcal{Q} \subset \mathbb{R}^{2}$, where $\mathbb{R}$ is the set of real numbers. Designing loitering patterns encompasses deciding the distribution of vehicles into teams, specifying the loitering curves for each team and the motion of vehicles along their respective loitering curves. Given a duration $T>0$, let $\gamma:[0, T] \rightarrow \mathbb{R}^{2}$ be a closed feasible curve for the Dubins vehicle or a closed 
Dubins path, i.e., $\gamma$ is a curve that is twice differentiable almost anywhere, $\left\|\gamma^{\prime}(t)\right\|=1$ for all $t \in[0, T]$, and the magnitude of the curvature of $\gamma$ is bounded above by $1 / \rho$, where $\rho>0$ is the minimum turning radius and $\gamma(0)=\gamma(1)$. The configuration of the Dubins vehicle traversing the curve $\gamma(t)$ will be denoted by $g_{\gamma}(t) \in \mathrm{SE}(2)$, where $\mathrm{SE}(2)$ is the special Euclidean group of dimension 2, i.e., $g_{\gamma}(t)=$ $\left(\gamma(t), \operatorname{ArcTan}\left(\gamma^{\prime}(t)\right)\right)$. Let the symbol $\mathbf{I} \in \mathrm{SE}(2)$ represent the identity element of the $\operatorname{SE}(2)$ group. Specifically, I will correspond to that state of the Dubins vehicle where it is positioned at the origin and its heading is aligned with the positive $\mathrm{X}$ axis. Let $\Gamma_{\rho}=\{\gamma \mid \gamma$ is a closed Dubins path $\}$. The loitering curves that are designed in this paper belong to $\Gamma_{\rho}$.

\section{B. The coverage problem}

Given $n$ vehicles, a team composition can be represented as $\left\{m_{1}, \ldots, m_{n}\right\}$, where $m_{i} \in \mathbb{N} \cup\{0\}$ and $\sum_{i=1}^{n} m_{i}=n$. Here $\mathbb{N}$ represents the set of natural numbers. In particular, if there are $\ell \leq n$ teams, then the team composition will be given by $\left\{m_{1}, \ldots, m_{\ell}, 0, \ldots, 0\right\}$. Let $\mathcal{M}(n)$ denote the set of all such possible team compositions. The idea is to partition $\mathcal{Q}$ into $\ell$ sub-regions such that each team is responsible for one sub-region. Given $\ell$ teams, let $\Lambda=\left(\gamma_{1}, \ldots, \gamma_{\ell}\right) \in \Gamma_{\rho}^{\ell}$ be a set of closed Dubins path for the teams. These curves will represent the loitering curves for the Dubins vehicle. In this paper we will be concerned with minimizing the worst case traveling time to any arbitrary (unknown) target point in $\mathcal{Q}$ by the closest Dubins vehicle. Since we constrain the vehicles to move at constant (unit) speed along the curves, one can prove by symmetry that the vehicles that are part of the same team are equally spaced along their common loitering curve and move in the same direction (i.e., clockwise/counter-clockwise). Therefore, given a region $\mathcal{Q}$ and a team composition $M=\left(m_{1}, \ldots, m_{\ell}, 0, \ldots, 0\right), \Lambda$ completely specifies the loitering pattern.

We now define the coverage cost associated with a given loitering pattern. Let $L_{\rho}: \operatorname{SE}(2) \times \mathbb{R}^{2} \rightarrow \mathbb{R}_{\geq 0}$ be the length of the shortest Dubins path from initial position and orientation, described by an element of $\operatorname{SE}(2)$, to a point $q \in \mathbb{R}^{2}$, where $\mathbb{R}$ is the set of real numbers and $\mathbb{R}_{\geq 0}$ is the set of non-negative real numbers. Recall that $L_{\rho}$ is continuous almost everywhere [11].

Definition 2.1 (Coverage cost): Given a region $\mathcal{Q}$, a team composition $M$, and a loitering pattern $\Lambda=\left(\gamma_{1}, \ldots, \gamma_{\ell}\right)$ with durations $\left(T_{1}, \ldots, T_{\ell}\right)$, define the coverage cost associated with the loitering pattern by

$$
\begin{aligned}
\mathcal{T}_{\mathcal{Q}, M}(\Lambda) & :=\sup _{q \in \mathcal{Q}} \min _{i \in\{1, \ldots, \ell\}} \\
& \sup _{s \in\left[0, \frac{T_{i}}{m_{i}}\right)} \min _{j \in\left\{1, \ldots, m_{i}\right\}} L_{\rho}\left(g_{\gamma_{i}}\left(s+\frac{(j-1) T_{i}}{m_{i}}\right), q\right) .
\end{aligned}
$$

The coverage cost gives the worst-case traveling time from any vehicle to any point in the region. In the rest of the paper, we will use coverage cost and cost interchangeably. The minimum cost associated with the given region $\mathcal{Q}$ and team composition $M$ is defined by

$$
\mathcal{T}_{\mathcal{Q}, M}^{*}:=\inf _{\Lambda \in \Gamma_{\rho}^{\ell}} \mathcal{T}_{\mathcal{Q}, M}(\Lambda) .
$$

In general, the optimal loitering patterns will have to be computed based on the shape of the region $\mathcal{Q}$. However, we will concentrate on circular loitering patterns; the rationale for doing so is that it (simplifies the problem and) allows us to provide algorithms and bounds that are independent of the particular shape of the environment. Furthermore, it seems unlikely that UAVs in the field will be able to compute optimal loitering patterns as their assigned regions change in real time; on the other hand, determining the location of the center, and the radius of a circular loitering patterns are much easier tasks.

For a given center $c \in \mathbb{R}^{2}$, radius $r>0$, let $\mathcal{O}(c, r)$ : $[0, T] \rightarrow \mathbb{R}^{2}$ represent a circle of radius $r$ with center c. A complete specification of a circular loitering curve would also involve specifying the direction of motion (i.e., clockwise or counter-clockwise) for the vehicles along the circle. However, in our problem setup, the coverage cost is not affected by the direction of motion as long as all the vehicles along the same circular loitering curve have the same direction of motion. Hence, we shall use $\mathcal{O}(c, r)$ along with an implicit and arbitrary assignment of direction of motion to it to also denote the corresponding circular loitering curve.

Since we will be concentrating only on circular curves, with a slight abuse of notation, we shall use $\Gamma_{\rho}$ to denote the set the circular curves with radii greater than or equal to $\rho$, i.e.,

$$
\Gamma_{\rho}=\{\mathcal{O}(c, r) \mid r \geq \rho\} .
$$

Accordingly, define a sub-minimum cost associated with the given region $\mathcal{Q}$ and team composition $M$ as:

$$
\widetilde{\mathcal{T}}_{\mathcal{Q}, M}^{*}:=\inf _{\Lambda \in \Gamma_{\rho}^{\ell}} \mathcal{T}_{\mathcal{Q}, M}(\Lambda),
$$

where the set of loitering curves is now a set of circular curves with centers at $c_{1}, \ldots, c_{\ell}$ and radii $r_{1}, \ldots, r_{\ell}$.

We are now ready to formulate the problem.

Problem 2.2 (Loitering problem): Given $n$ Dubins vehicles with known team composition for a region $\mathcal{Q}$, design circular loitering patterns that minimize the cost function given by equation (1).

\section{Geometric notions}

We need to define a few more notations and concepts. Consider a point $c \in \mathbb{R}^{2}$ and $r>0$. For a region $U \subset$ $\mathbb{R}^{2}$, let $\operatorname{Ann}(c, U)$ be the smallest annulus centered at $c$ and containing $U$, i.e., $\operatorname{Ann}(c, U)=\cup_{q \in U} \mathcal{O}(c,\|q-c\|)$. Let $\mathrm{B}(c, r)$ be the closed ball of radius $r$ and centered at $c$. Let $\operatorname{EB}(c, U)$ be the smallest closed ball enclosing $U$ centered at $c$, i.e., $\mathrm{EB}(c, U)=\mathrm{B}\left(c, \sup _{q \in U}\|q-c\|\right)$, where $\|$.$\| represents the Euclidean norm. Let \operatorname{CR}(U)$ be the circumradius and $\operatorname{CC}(U)$ be the circumcenter of $\mathrm{U}$. Finally, given a set of angles $\theta \in[0,2 \pi), \triangle \theta \in[0,2 \pi]$, let $\mathrm{S}(c, r, \theta, \triangle \theta)$ be the sector traced by a segment of length $r$ and fixed at $c$ as it rotates from the angle $\theta$ to the angle 


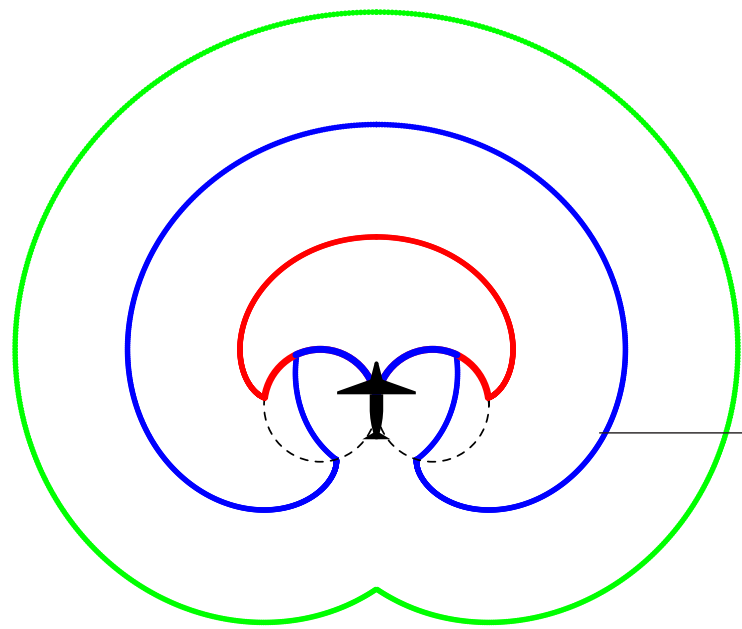

Fig. 1. Reachable sets $\mathcal{R}_{\mathbf{I}}(t)$ for the Dubins vehicle for $t=3 \rho, 5 \rho$ and $7 \rho$.

$\theta+\triangle \theta$ in the counter-clockwise direction. With this notation, $\mathrm{B}(c, r)=\mathrm{S}(c, r, 0,2 \pi)$.

\section{A Dubins REAChABLE SET COVERING PROBLEM}

In this section we state some properties of the Dubins reachable set which shall be useful in the due course of the paper. Then, we introduce a "set covering problem" that will play a key role in the design of efficient loitering patterns.

\section{A. Dubins reachable set}

Given $t \geq 0$ and a Dubins state $h \in \mathrm{SE}(2)$, let $\mathcal{R}_{h}(t)$ denote the reachable set of the Dubins vehicle in time $t$ starting from state $h$, i.e.,

$$
\mathcal{R}_{h}(t)=\left\{q \in \mathbb{R}^{2} \mid L_{\rho}(h, q) \leq t\right\} .
$$

Reachable sets for the Dubins vehicle are shown in Fig. 1. The boundary of the reachable sets consist of arcs of circle involutes and arcs of epicycloids (for further details on these families of curves see, e.g., [12]). We shall also use a slightly truncated version of $\mathcal{R}_{h}(t)$ for sufficiently large $t$. We will denote this set by $\widetilde{\mathcal{R}}_{h}(t)$. For the sake of clarity we explain the construction of $\widetilde{\mathcal{R}}_{\mathbf{I}}(t)$ from $\mathcal{R}_{\mathbf{I}}(t)$ with the help of Fig. 2 as follows: Consider the axis that is perpendicular to the heading of the Dubins vehicle. Let this axis intersect the boundary of $\mathcal{R}_{\mathbf{I}}(t)$ at $P_{L}(t)$ and $P_{R}(t)$. Let $P_{B}(t)$ be the furthest point that lies exactly behind the Dubins vehicle. Let $H_{L}(t)$ be the half-plane generated by the line passing through $P_{L}(t)$ and $P_{B}(t)$ that does not contain the origin. Similarly, let $H_{R}(t)$ be the half-plane generated by the line passing through $P_{R}(t)$ and $P_{B}(t)$ that does not contain the origin. Then $\widetilde{\mathcal{R}}_{\mathbf{I}}(t)=\mathcal{R}_{\mathbf{I}}(t) \backslash\left(H_{L}(t) \cap H_{R}(t)\right)$. It follows from the above construction that $\widetilde{\mathcal{R}}_{h}(t)$ is an inner approximation of $\mathcal{R}_{\mathbf{I}}(t)$. Moreover, as observed in Figure 2, the approximation is fairly tight.

Using the definition of the reachable sets and planar geometry, one can prove that the following properties hold true for any $h \in \mathrm{SE}(2)$.

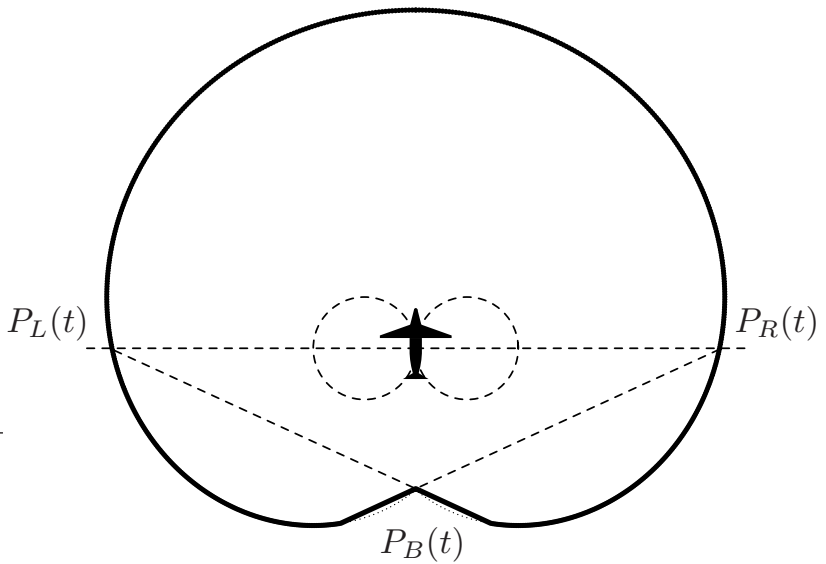

Fig. 2. Truncation of $\mathcal{R}_{h}(t)$ to form $\widetilde{\mathcal{R}}_{h}(t)$.

(P1) $\mathcal{R}_{h}(t)$ is a monotonic function in $t$, i.e., $\mathcal{R}_{h}\left(t^{\prime}\right) \subseteq$ $\mathcal{R}_{h}(t)$ for $t^{\prime} \leq t$.

There exist constants $\kappa_{1} \in[5.7,5.8]$ and $\kappa_{2} \in[6.5,6.6]$ such that

(P2) $\mathcal{R}_{h}(t)$ is a simply connected set for all $t \in \mathbb{R}_{\geq 0} \backslash$ $\left[\kappa_{1} \rho, \kappa_{2} \rho\right]$, and

(P3) For all $t \geq \kappa_{2} \rho, \widetilde{\mathcal{R}}_{h}(t)$ is star-shaped ${ }^{1}$ and the kernel ${ }^{2}$ of $\widetilde{\mathcal{R}}_{h}(t)$ is the set of points that lie on the axis which is perpendicular to the heading direction of the vehicle at $h$.

\section{B. A set covering problem}

In this section, we formulate a set covering problem for the Dubins reachable set in the form of an optimization problem. The idea is to find the smallest reachable set that contains a given subset of the environment under some constraints on the relative position between the reachable set and the subset of the environment. The motivation for this will become clearer in subsequent sections, where we assign subsets of the environment to each vehicle and then use the set covering problem to design circular loitering patterns. For the purpose of the optimization problem, we will tacitly replace $\mathcal{R}_{h}(t)$ by its tight inner approximation $\widetilde{\mathcal{R}}_{h}(t)$ for $t \geq \kappa_{2} \rho$.

For a given positive integer $m$ and $t \geqslant 0$, we state the following optimization problem with variables $r, \lambda$ and $\theta$.

$$
\begin{aligned}
\operatorname{maximize} & r, \\
\text { subj. to } & r \geqslant 0, \\
& \lambda \geqslant \rho, \\
& 0 \leqslant \theta \leqslant 2 \pi, \\
& \mathrm{S}\left((0, \lambda), r, \theta, \frac{2 \pi}{m}\right) \subseteq \mathcal{R}_{\mathbf{I}}(t) .
\end{aligned}
$$

For a fixed $m$, we associate the solution of this optimization problem with functions $\mathrm{R}_{m}, \Lambda_{m}, \Theta_{m}$ :

\footnotetext{
${ }^{1}$ A region $U$ is called star-shaped if there is a point $a \in U$ such that the line segment $\overline{a b}$ is contained in $U$ for all $b \in U$. Here $\overline{a b}=\{t a+(1-$ $t) b \mid t \in[0,1]\}$. We then say that $U$ is star-shaped with respect to $a$.

${ }^{2}$ The kernel of a star-shaped region $U$ is the set of points from which the entire set $U$ is visible
} 
$\mathbb{R}_{\geq 0} \rightarrow \mathbb{R}_{\geq 0}, m=1,2, \ldots$, which are defined so that $\left(\mathbf{R}_{m}(t), \Lambda_{m}(t), \Theta_{m}(t)\right)$ is the value of $(r, \lambda, \theta)$ at optimality for the above optimization problem.

Geometrically, for a fixed $m$ and $t$, a solution to the optimization problem gives the specifications of the largest sector extending an angle $\frac{2 \pi}{m}$ at the origin such that it is contained inside $\mathcal{R}_{\mathbf{I}}(t)$ and its center lies on the $Y$ axis and at least at a distance of $\rho$ from the origin. Specifically, the radius of such a sector is given by $\mathrm{R}_{m}(t)$, its center is at $\Lambda_{m}(t)$ and it is contained between the rays making angles $\Theta_{m}(t)$ and $\Theta_{m}(t)+\frac{2 \pi}{m}$ with the $X$ axis. In particular, $\mathrm{R}_{1}(t)$ denotes the radius of the largest disk contained inside $\mathcal{R}_{\mathbf{I}}(t)$ and whose center lies on the $Y$ axis and at least $\rho$ distance from the origin.

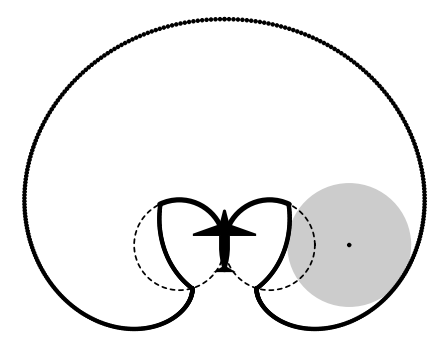

Fig. 3. Finding the value $\mathrm{R}_{1}(5 \rho)$.

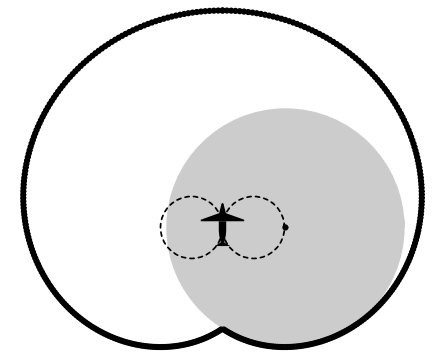

Fig. 4. Finding the value $\mathrm{R}_{1}(7 \rho)$.

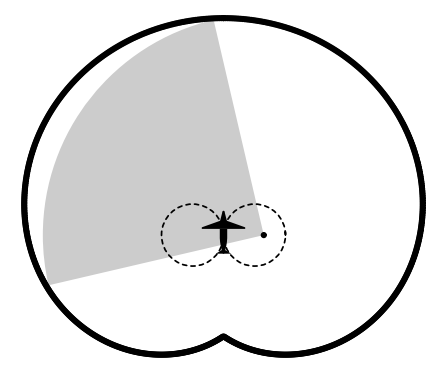

Fig. 5. Finding the value $\mathrm{R}_{4}(7 \rho)$.

One can show that, at fixed $m$, the function $t \mapsto \mathrm{R}_{m}(t)$ is a strictly increasing function in $t$. This is also illustrated in Figs. 6 and 7, where we have plotted $\mathrm{R}_{1}(t)$ vs. $t$ and $\mathrm{R}_{2}(t)$ vs. $t$ respectively for $\rho=1$. Hence, the inverse function $\mathbf{R}_{m}^{-1}$ is also well defined

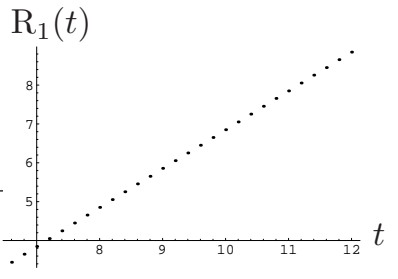

Fig. 6. Plot of $\mathrm{R}_{1}(t)$ vs. $t$ for $\rho=1$.

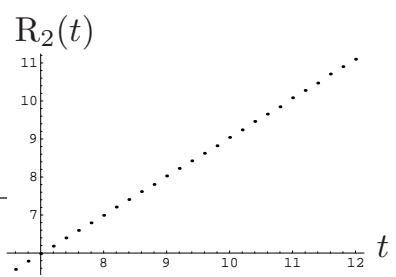

Fig. 7. Plot of $\mathrm{R}_{2}(t)$ vs. $t$ for $\rho=1$.

\section{THE SINGLE VEHICLE CASE}

In this section we concentrate our attention on the case when $M=(1)$, i.e., only one vehicle is assigned the task to service the region $\mathcal{Q}$. For brevity in notation for this section, we shall drop 1 from the subscript of the notations for the various cost functions: $\mathcal{T}, \mathcal{T}^{*}$ and $\widetilde{\mathcal{T}}^{*}$.

Theorem 4.1 (Equivalence by rotation): For a region $\mathcal{Q}$, $\rho>0, c \in \mathbb{R}^{2}$ and $r \geq \rho$,

(i) $\mathcal{T}_{\mathcal{Q}}(\mathcal{O}(c, r))=\mathcal{T}_{\operatorname{Ann}(c, \mathcal{Q})}(\mathcal{O}(c, r))$,

(ii) $\mathcal{T}_{\mathcal{Q}}(\mathcal{O}(c, r))$ is the minimum $t \geqslant 0$ such that $\mathcal{R}_{\mathbf{I}}(t) \supseteq$ $\cup_{q \in \mathcal{Q}} \mathcal{O}((0, r),\|c-q\|)$.

In addition, if $\mathcal{T}_{\mathcal{Q}}(\mathcal{O}(c, r)) \in \mathbb{R}_{\geq 0} \backslash\left[\kappa_{1} \rho, \kappa_{2} \rho\right]$, then

$$
\mathcal{T}_{\mathcal{Q}}(\mathcal{O}(c, r))=\mathcal{T}_{\mathrm{EB}(c, \mathcal{Q})}(\mathcal{O}(c, r)) .
$$

We are now ready to state the main result of this section.

Theorem 4.2 (An optimal circular loitering curve):

Given a region $\mathcal{Q}$ for which $\operatorname{CR}(\mathcal{Q}) \in \mathbb{R}_{\geq 0}$ । $\left[\mathrm{R}_{1}\left(\kappa_{1} \rho\right), \mathrm{R}_{1}\left(\kappa_{2} \rho\right)\right]$, the circle of radius $\Lambda_{1}\left(\mathrm{R}_{1}^{-1}(\mathrm{CR}(\mathcal{Q}))\right)$ with center at $\mathrm{CC}(\mathcal{Q})$ is an optimal circular loitering curve over $\mathcal{Q}$, and the corresponding coverage cost is given by

$$
\widetilde{\mathcal{T}}_{\mathcal{Q}}^{*}=\mathrm{R}_{1}^{-1}(\mathrm{CR}(\mathcal{Q})) .
$$

Remark 4.3 (Circular loitering patterns are optimal): Although we have been restricting our attention on circular loitering curves, one can prove that, for the single vehicle case, an optimal circular loitering curve is also an optimal loitering curve, i.e.,

$$
\mathcal{T}_{\mathcal{Q}}^{*}=\widetilde{\mathcal{T}}_{\mathcal{Q}}^{*}=\mathrm{R}_{1}^{-1}(\mathrm{CR}(\mathcal{Q})) .
$$

\section{THE SINGLE TEAM CASE}

In this section we design a loitering circle for a team of $n$ Dubins vehicles servicing the region $\mathcal{Q}$, i.e., $M=$ $(n, 0, \ldots, 0)$. For brevity in notation, we shall denote this team composition by $M=(n)$. By symmetry, the $n$ vehicles will be placed at an angular distance of $\frac{2 \pi}{n}$ from each other. We now introduce the concept of Dubins Voronoi Partition for vehicles moving along a circular loitering curve $\mathcal{O}(c, r)$. 
Let $g=\left\{g_{1}, \ldots, g_{n}\right\} \in \mathrm{SE}(2)^{n}$ be a state of the $n$ equally spaced Dubins vehicles loitering along the curve $\mathcal{O}(c, r)$. We note here that, due to the symmetrical placement of the vehicles along the loitering curve, the set $g$ is completely defined by specifying any one of its elements. We define the Dubins Voronoi cell generated by $g_{i}$ by

$$
\begin{aligned}
& V\left(g_{i}, c, r, n\right) \\
= & \left\{q \in \mathbb{R}^{2} \mid L_{\rho}\left(g_{i}, q\right) \leq L_{\rho}\left(g_{j}, q\right) \text { for all } j \in\{1, \ldots, n\}\right\} .
\end{aligned}
$$

Theorem 5.1 (Equivalence by rotation): For a region $\mathcal{Q}$, $\rho>0, c \in \mathbb{R}^{2}, r \geq \rho$ and $n>1$,

(i) $\mathcal{T}_{\mathcal{Q}, n}(\mathcal{O}(c, r))=\mathcal{T}_{\text {Ann }(c, \mathcal{Q}), n}(\mathcal{O}(c, r))$,

(ii) Pick any $h \in \mathrm{SE}(2)$ along the circular loitering curve $\mathcal{O}(c, r)$. Then, $\mathcal{T}_{\mathcal{Q}, n}(\mathcal{O}(c, r))$ is the minimum $t \geqslant 0$ such that $\mathcal{R}_{h}(t) \supseteq V(h, c, r, n) \cap \operatorname{Ann}(c, \mathcal{Q})$.

Theorem 5.1 suggests how to compute the optimal circular trajectory for a team of Dubins vehicles by converting it into an optimization problem for a single vehicle. However, solving this optimization problem requires the knowledge of the shape of Dubins Voronoi partitions. Even though there is an element of rotational symmetry in our case, the shapes of the Dubins Voronoi partition (e.g., see Figure 8) are not easy enough to lend themselves to analysis. Hence, we shall approximate the Voronoi partitions by sectors as shown in Figure 9. This approximation helps in deriving upper bounds on the cost function.

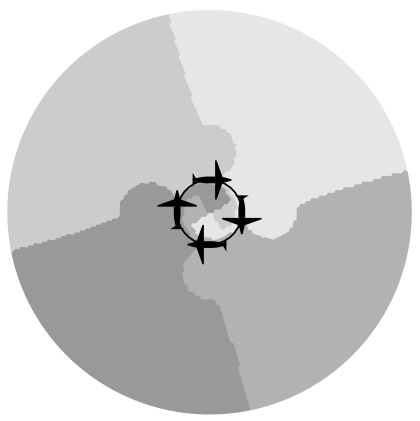

Fig. 8. Dubins Voronoi partition for 4 vehicles loitering symmetrically along a common circular curve.

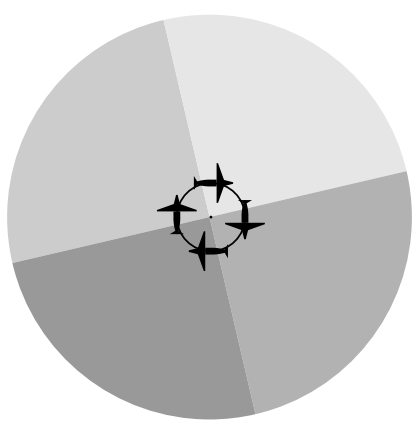

Fig. 9. Approximation of Dubins Voronoi partition by sectors.

We are now ready to state the main result of the section.
Theorem 5.2: (An upper bound on the coverage cost for a single team in large environments) Given a region $\mathcal{Q}$ for which $\mathrm{CR}(\mathcal{Q}) \geq \mathrm{R}_{n}\left(\kappa_{2} \rho\right)$, the circle of radius $\Lambda_{n}\left(\mathrm{R}_{n}^{-1}(\mathrm{CR}(\mathcal{Q}))\right)$ with center at $\mathrm{CC}(\mathcal{Q})$ is an approximation to the optimal circular loitering curve over $\mathcal{Q}$, and the corresponding upper bound on the coverage cost is given by

$$
\widetilde{\mathcal{T}}_{\mathcal{Q}, n}^{*} \leq \mathrm{R}_{n}^{-1}(\mathrm{CR}(\mathcal{Q})) .
$$

Remark 5.3: The bound obtained in Theorem 5.2 is tightest among the bounds possible by approximations of Dubins Voronoi partitions for vehicles moving along circular curves by sectors of circles.

\section{The MULTiPle UNIFORM TEAM CASE}

In this section we consider the multiple team case, concentrating on the case when the teams have uniform composition. A group of $n$ vehicles comprising of $\ell$ teams is said to have uniform team composition if $n$ is a multiple of $\ell$ and the team composition is of the form $\left(\frac{n}{l}, \ldots, \frac{n}{l}, 0, \ldots, 0\right)$. We shall show that, for a sufficiently large and convex region $\mathcal{Q}$, an upper bound on the cost of coverage by the $l$ team of loitering Dubins vehicles can be obtained by solving a related disk covering problem.

We first briefly describe the disk-covering problem or, more precisely, the version of the disk covering problem that is relevant for our purposes here. In our context, the disk covering problem can be stated as follows: given a convex region $\mathcal{Q}$ and an integer $\ell$, find the smallest real number $\operatorname{RDC}_{\mathcal{Q}}(\ell)$ and a set of locations $\left\{c_{1}, \ldots, c_{\ell}\right\}$ such that the $\ell$ disks, each of radius $\operatorname{RDC}_{\mathcal{Q}}(\ell)$ and centered at $\left\{c_{1}, \ldots, c_{\ell}\right\}$ cover $\mathcal{Q}$, that is, $\mathcal{Q} \subset \cup_{i \in\{1, \ldots, \ell\}} \mathrm{B}\left(c_{i}, \operatorname{RDC}_{\mathcal{Q}}(\ell)\right)$. We shall refer to $\left(\operatorname{RDC}_{\mathcal{Q}}(\ell),\left\{c_{1}, \ldots, c_{\ell}\right\}\right)$ as the solution to the disk covering problem for $\mathcal{Q}$.

Disk covering problems have a long and beautiful history [14]. Many variants of the problem (e.g., geometric minimum disk cover problem) find their applications in numerous engineering applications (e.g., localization in sensor networks).

In [13] distributed algorithms were designed to solve the disk covering problem via a dynamical systems approach. Specifically, the paper proposes the move toward the furthest and move toward the circumcenter algorithms for a group of $\ell$ mobile robots. In the move toward the furthest algorithm, each "disk center" moves towards the furthest vertex of its Voronoi cell (inside the Voronoi partition generated by all "disk centers"). In the 'move toward the circumcenter' algorithm, each disk-center moves toward the circumcenter of its Voronoi cell. In both algorithms the Voronoi partition is continuously updated as the disk centers move. Asymptotically, an execution of one of these two algorithms computes a locally optimal solution to the disk covering problem in the sense that the location of these robots correspond to the centers $c_{1}, \ldots, c_{\ell}$ and the largest of the circumradii of the Voronoi partitions corresponds to $\operatorname{RDC}_{\mathcal{Q}}(\ell)$. Moreover, these distributed control laws can be implemented as local interactions between the disk centers. In our setting, this would imply that this would require interaction only between 

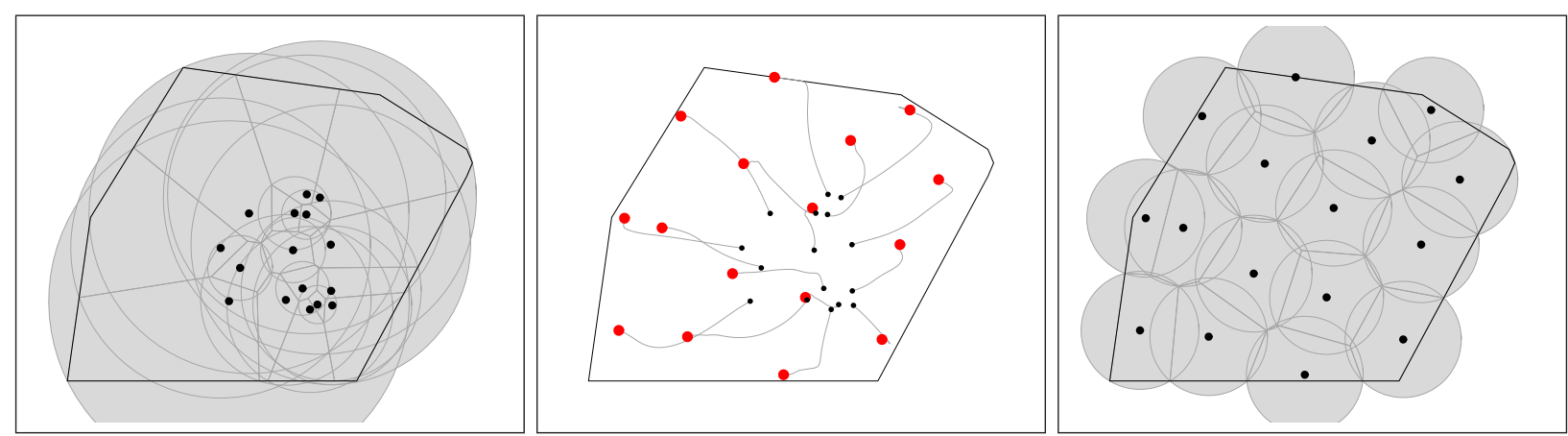

Fig. 10. "Move-toward-the-circumcenter" algorithm for 16 disks in a convex polygonal domain. The left (respectively, right) figure illustrates the initial (respectively, final) locations and Voronoi partition. The central figure illustrates the network evolution. After 20 sec., the disk radius is approximately $0.43273 \mathrm{~m}$. Simulations taken from [13].

neighboring teams of vehicles, i.e., teams whose center of rotations are Voronoi neighbors. An execution of the move toward the circumcenter algorithm is illustrated in Figure 10.

We now state the following result which gives an upper bound on the coverage cost for multiple uniform teams of loitering Dubins vehicles.

Theorem 6.1: Consider a group of $n$ Dubins vehicles divided into $\ell$ teams of uniform composition loitering in a convex region $\mathcal{Q}$. Let $\left(\operatorname{RDC}_{\mathcal{Q}}(\ell),\left\{c_{1}, \ldots, c_{\ell}\right\}\right)$ be the solution to the disk covering problem for $\mathcal{Q}$. If $\operatorname{Area}(\mathcal{Q}) \geq$ $\ell \pi \mathrm{R}_{\frac{n}{\ell}}^{2}\left(\kappa_{2} \rho\right)$, then

$$
\widetilde{\mathcal{T}}_{\mathcal{Q},\left(\frac{n}{\ell}, \ldots, \frac{n}{\ell}, 0, \ldots, 0\right)}^{*} \leq \mathrm{R}_{\frac{n}{\ell}}^{-1}\left(\operatorname{RDC}_{\mathcal{Q}}(\ell)\right) .
$$

Moreover, the loitering pattern which achieves this upper bound is the set of circular curves, each of radius $\Lambda_{\frac{n}{\ell}}\left(\mathrm{R}_{\frac{n}{\ell}}^{-1}\left(\operatorname{RDC}_{\mathcal{Q}}(\ell)\right)\right)$, and with centers at $\left\{c_{1}, \ldots, c_{\ell}\right\}$.

Using the control algorithms from [13], one can design a computational approach to computing loitering patterns as follows:

(i) Partition the environment into Voronoi partitions generated by virtual centers.

(ii) Move the virtual centers in such a way as to solve a minimum-radius disk-covering problem

(iii) Designing efficient loitering patterns for each team in its corresponding Voronoi cell.

\section{CONCLUSION}

In this paper, we considered the coverage problem for loitering Dubins vehicles. We have characterized the configuration of the vehicles at the appearance of new targets in terms of Dubins paths, that we call loitering patterns. We defined the coverage cost to be the worst-case traveling time from any vehicle to any point in the region. Optimal circular loitering for a single vehicle and efficient circular loitering for a single team of vehicles were characterized. Finally, by establishing an analogy to the disk-covering problem, we proposed a computational approach to characterize efficient loitering patterns for multiple uniform teams.

This paper leaves numerous important extensions open for further research. One needs to study the functions $\mathrm{R}_{n}^{-1}$ to derive closed form expression for the bounds derived in this paper. It would be interesting to consider the coverage problem for other meaningful cost functions. The problem of multi non-uniform team of vehicles is also important. Determining the ideal team composition for a given region provides an exciting challenge too.

\section{REFERENCES}

[1] C. Schumacher, P. R. Chandler, S. J. Rasmussen, and D. Walker, "Task allocation for wide area search munitions with variable path length," in American Control Conference, (Denver, CO), pp. 3472-3477, 2003.

[2] R. W. Beard, T. W. McLain, M. A. Goodrich, and E. P. Anderson, "Coordinated target assignment and intercept for unmanned air vehicles," IEEE Transactions on Robotics and Automation, vol. 18, no. 6, pp. 911-922, 2002.

[3] A. Richards, J. Bellingham, M. Tillerson, and J. How, "Coordination and control of multiple UAVs," in AIAA Conf. on Guidance, Navigation and Control, (Monterey, CA), Aug. 2002.

[4] A. E. Gil, S. Ganapathy, K. M. Passino, and A. Sparks, "Cooperative scheduling of tasks for networked uninhabited autonomous vehicles," in IEEE Conf. on Decision and Control, (Maui, Hawaii), pp. 522-527, Dec. 2003.

[5] J. Cortés, S. Martínez, T. Karatas, and F. Bullo, "Coverage control for mobile sensing networks," IEEE Transactions on Robotics and Automation, vol. 20, no. 2, pp. 243-255, 2004.

[6] K. Savla, E. Frazzoli, and F. Bullo, "Traveling Salesperson Problems for the Dubins vehicle," IEEE Transactions on Automatic Control, 2007. (Submitted Jun 2006) To appear.

[7] L. E. Dubins, "On curves of minimal length with a constraint on average curvature and with prescribed initial and terminal positions and tangents," American Journal of Mathematics, vol. 79, pp. 497516, 1957.

[8] A. Ahmadzadeh, J. Keller, G. Pappas, A. Jadbabaie, and V. Kumar, "Multi-UAV cooperative surveillance with spatio-temporal specifications," in IEEE Conf. on Decision and Control, (San Diego, CA), pp. 5293-5298, 2006.

[9] S. Rathinam, M. Zennaro, T. Mak, and R. Sengupta, "An architecture for UAV team control," in IFAC Symposium on Intelligent Autonomous Vehicles, (Lisbon, Portugal), July 2004.

[10] K. Savla, Multi UAV Systems with Motion and Communication Constraints. PhD thesis, Electrical and Computer Engineering Department, University of California at Santa Barbara, Santa Barbara, Aug. 2007. Available electronically at http://ccdc. mee. ucsb.edu.

[11] J. J. Enright, E. Frazzoli, K. Savla, and F. Bullo, "On multiple UAV routing with stochastic targets: performance bounds and algorithms,' in AIAA Conf. on Guidance, Navigation and Control, Aug. 2005. Electronic Proceedings.

[12] J. Lawrence, A catalog of special plane curves. New York: Dover Publications, 1972.

[13] J. Cortés and F. Bullo, "Coordination and geometric optimization via distributed dynamical systems," SIAM Journal on Control and Optimization, vol. 44, no. 5, pp. 1543-1574, 2005.

[14] R. Kershner, "The number of circles covering a set," American Journal of Mathematics, vol. 61, no. 3, pp. 665-671, 1939. 